\title{
SIMILARITY SOLUTIONS OF A CLASS OF LAMINAR THREE-DIMENSIONAL BOUNDARY LAYER EQUATIONS OF POWER LAW FLUIDS
}

\author{
TSUNG Yen NA \\ Department of Mechanical Engineering, University of Michigan, Dearborn, Michigan \\ and \\ ARTHUR G. HANSEN \\ College of Engineering, Georgia Institute of Technology, Atlanta, Georgia
}

\begin{abstract}
An analysis of the possibility of finding similarity solutions to the three-dimensional, steady, incompressible, boundary layer equations in rectangular coordinates for a power law fluid is investigated. It is found that, in general, the two components of the mainstream flow must differ by at most a multiplicative constant and that these components are powers or exponentials of the $x^{\prime}$-coordinate.

By assuming small cross-flows, the cross flow component may be generalized and found to be representable by a polynomial in the through flow variable, $x^{\prime}$.
\end{abstract}

\section{NOTATION}

$A, a_{i} \quad$ arbitrary constants

$C_{1} \ldots C_{8}$ arbitrary constants

$F, G \quad$ dependent variables in the transformed ordinary differential equations

$i, j \quad$ arbitrary constants

$L \quad$ a characteristic length

$m, n \quad$ parameters in the mathematical model of a power-law fluid

Ren Reynolds number, equation (4)

$s \quad$ an arbitrary constant

$u \quad$ velocity component in the boundary layer along the $x$-axis

$U \quad$ velocity component in the mainflow along the $x$-axis

$V_{0} \quad$ a characteristic velocity

$v \quad$ velocity component in the boundary layer along the $y$-axis

$w \quad$ velocity component in the boundary layer along the $z$-axis

$W \quad$ velocity component in the mainflow along the $z$-axis

$x, y \quad$ Cartesian coordinates

$\alpha_{1} \ldots a_{6} \quad$ arbitrary constants

$\alpha \quad \alpha_{4} / \alpha_{1}$

$\beta_{1} \ldots \beta_{6} \quad$ arbitrary constants

$\beta \quad$ arbitrary constant

$\rho \quad$ density of the fluid

$\psi \quad$ a mathematical function

$\eta, \xi \quad$ independent variables in the transformed ordinary differential equations

$\theta \quad$ deflection angle of boundary layer streamlines

$\tau \quad$ the stress tensor

$\Delta \quad$ the rate of deformation tensor

$\tau_{y x}, \tau_{y z}$ the two non-vanishing components of the stress tensor 


\section{INTRODUCTION}

THE boundary layer flows for non-Newtonian fluids of the Ostwald-de Waele model have been discussed recently in a number of papers [1-4]. Except for the work of Schowalter [4], all papers refer to two-dimensional flows. Schowalter considered the laminar, three-dimensional incompressible boundary layer equations with a Cartesian coordinate system. The conclusion was that similarity solutions exist only for the case of both mainstream velocity components $U$ and $W$ being constants. The restrictions are severe.

In the present paper, a similar problem is considered except that all flow quantities are independent of the $z^{\prime}$-coordinate, as shown in Fig. 1. Such flows are characterized by the fact that their streamlines form a system of "translates". That is, the entire streamline pattern can be obtained by translating any particular streamline parallel to the leading edge of the surface [5]. It is hoped that by omitting dependence of flow quantities in one direction, more qualitative information may be obtained on the characteristics of the three-dimensional boundary layer flows of power-law non-Newtonian fluids.

\section{THE BASIC EQUATIONS}

The power-law Ostwald-de Waele model has been found to be remarkably versatile and useful in representing flow behavior of many non-Newtonian fluids over quite a wide range of shearing rate. Mathematically, it can be represented in the form

$$
\overline{\bar{\tau}}=-\left\{m\left|\sqrt{ }\left(\frac{1}{2} \overline{\bar{\Delta}}: \overline{\bar{\Delta}}\right)\right|^{n-1}\right\} \overline{\bar{\Delta}}
$$

where $\overline{\bar{\tau}}$ and $\overline{\bar{\Delta}}$ are the stress tensor and the rate of deformation tensor, respectively; and $m$ and $n$ are physical constants different for different fluids which can be determined experimentally. Under the boundary layer assumptions, the only two non-vanishing components are

$$
\tau_{y x}=-m\left\{\left[\left(\frac{\partial u^{\prime}}{\partial y^{\prime}}\right)^{2}+\left(\frac{\partial w^{\prime}}{\partial y^{\prime}}\right)^{2}\right]^{\frac{n-1}{2}} \frac{\partial u^{\prime}}{\partial y^{\prime}}\right\}
$$

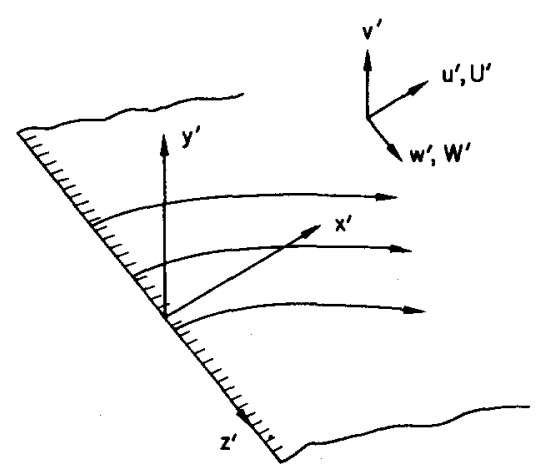

FIG. 1. Coordinate system for flow over plate. 
and

$$
\tau_{y z}=-m\left\{\left[\left(\frac{\partial u^{\prime}}{\partial y^{\prime}}\right)^{2}+\left(\frac{\partial w^{\prime}}{\partial y^{\prime}}\right)^{2}\right]^{\frac{n-1}{2}} \frac{\partial w^{\prime}}{\partial y^{\prime}}\right\}
$$

where the absolute sign has been dropped since both terms within the sign are positive. Using this "equation of state", the steady, incompressible, laminar boundary layer equations over a surface oriented in Fig. 1 are:

Continuity

$$
\frac{\partial u^{\prime}}{\partial x^{\prime}}+\frac{\partial v^{\prime}}{\partial y^{\prime}}=0
$$

Momentum

$$
\begin{aligned}
& u^{\prime} \frac{\partial u^{\prime}}{\partial x^{\prime}}+v^{\prime} \frac{\partial u^{\prime}}{\partial y^{\prime}}=\frac{m}{\rho} \frac{\partial}{\partial y^{\prime}}\left\{\left[\left(\frac{\partial u^{\prime}}{\partial y^{\prime}}\right)^{2}+\left(\frac{\partial w^{\prime}}{\partial y^{\prime}}\right)^{2}\right]^{\frac{n-1}{2}} \frac{\partial u^{\prime}}{\partial y^{\prime}}\right\}+U^{\prime} \frac{\mathrm{d} U^{\prime}}{\mathrm{d} x^{\prime}} \\
& u^{\prime} \frac{\partial w^{\prime}}{\partial x^{\prime}}+v^{\prime} \frac{\partial w^{\prime}}{\partial y^{\prime}}=\frac{m}{\rho} \frac{\partial}{\partial y^{\prime}}\left\{\left[\left(\frac{\partial u^{\prime}}{\partial y^{\prime}}\right)^{2}+\left(\frac{\partial w^{\prime}}{\partial y^{\prime}}\right)^{2}\right]^{\frac{n-1}{2}} \frac{\partial w^{\prime}}{\partial y^{\prime}}\right\}+U^{\prime} \frac{\mathrm{d} W^{\prime}}{\mathrm{d} x^{\prime}}
\end{aligned}
$$

The boundary conditions for the system of equations are

$$
\begin{array}{ll}
y^{\prime}=0: & u^{\prime}=v^{\prime}=w^{\prime}=0 \\
y^{\prime}=\infty: & u^{\prime}=U^{\prime}\left(x^{\prime}\right), \quad w^{\prime}=W^{\prime}\left(x^{\prime}\right)
\end{array}
$$

The flow problem is quasi-two-dimensional in nature since the velocity components are independent of the $z$-coordinates. This point is discussed in detail by Hansen and Herzig [5].

Equations (1), (2) and (3) may be put in dimensionless form as follows:

$$
\begin{aligned}
u=\frac{u^{\prime}}{U_{0}}, & v=\frac{v^{\prime}}{U_{0}} \operatorname{Ren}^{\frac{1}{n+1}}, & w=\frac{w^{\prime}}{U_{0}}, \quad \bar{U}=\frac{U^{\prime}}{U_{0}} \\
W=\frac{W^{\prime}}{U_{0}}, & x=\frac{x^{\prime}}{L}, & y=\frac{y^{\prime}}{L} \operatorname{Ren}^{\frac{1}{n+1}}
\end{aligned}
$$

where

$$
\operatorname{Ren}=\frac{\rho U_{0}^{2-n} L^{n}}{m}
$$

Then, we obtain:

Continuity equation:

$$
\frac{\partial u}{\partial x}+\frac{\partial v}{\partial y}=0
$$


Momentum equation:

$$
\begin{aligned}
& u \frac{\partial u}{\partial x}+v \frac{\partial u}{\partial y}=\frac{\partial}{\partial y}\left\{\left[\left(\frac{\partial u}{\partial y}\right)^{2}+\left(\frac{\partial w}{\partial y}\right)^{2}\right]^{\frac{n-1}{2}} \frac{\partial u}{\partial y}\right\}+U \frac{\mathrm{d} U}{\mathrm{~d} x} \\
& u \frac{\partial w}{\partial x}+v \frac{\partial w}{\partial y}=\frac{\partial}{\partial y}\left\{\left[\left(\frac{\partial u}{\partial y}\right)^{2}+\left(\frac{\partial w}{\partial y}\right)^{2}\right]^{\frac{n-1}{2}} \frac{\partial w}{\partial y}\right\}+U \frac{\mathrm{d} W}{\mathrm{~d} x}
\end{aligned}
$$

with the boundary conditions

$$
\begin{array}{ll}
y=0: & u=v=w=0 \\
y=\infty: & u=U(x), \quad w=W(x)
\end{array}
$$

The equation of continuity can be satisfied identically by introducing a function,* $\psi$, which gives

$$
u=\frac{\partial \psi}{\partial y} \quad \text { and } \quad v=-\frac{\partial \psi}{\partial x}
$$

Equations (5), (6) and (7) then become

$$
\begin{gathered}
\frac{\partial \psi}{\partial y} \frac{\partial^{2} \psi}{\partial x \partial y}-\frac{\partial \psi}{\partial x} \frac{\partial^{2} \psi}{\partial y^{2}}=\frac{\partial}{\partial y}\left\{\left[\left(\frac{\partial^{2} \psi}{\partial y^{2}}\right)^{2}+\left(\frac{\partial w}{\partial y}\right)^{2}\right]^{\frac{n-1}{2}} \frac{\partial^{2} \psi}{\partial y^{2}}\right\}+U \frac{\mathrm{d} U}{\mathrm{~d} x} \\
\frac{\partial \psi}{\partial y} \frac{\partial w}{\partial x}-\frac{\partial \psi}{\partial x} \frac{\partial w}{\partial y}=\frac{\partial}{\partial y}\left\{\left[\left(\frac{\partial^{2} \psi}{\partial y^{2}}\right)^{2}+\left(\frac{\partial w}{\partial y}\right)^{2}\right]^{\frac{n-1}{2}} \frac{\partial w}{\partial y}\right\}+U \frac{\mathrm{d} W}{\mathrm{~d} x}
\end{gathered}
$$

with the boundary conditions

$$
\begin{array}{ll}
y=0: & \frac{\partial \psi}{\partial x}=\frac{\partial \psi}{\partial y}=\quad w=0 \\
y=\infty: & \frac{\partial \psi}{\partial y}=U(x), \quad w=W(x)
\end{array}
$$

A group-theoretic analysis is employed in the next section to find the form of $U(x)$ and $W(x)$ for which similarity solutions will exist.

\section{GROUP-THEORETIC ANALYSIS}

Similarity analysis by the group-theoretic method is based on concepts derived from the theory of transformation groups. This method was first introduced by Birkhoff [6] and Morgan [7] and is discussed in detail in [8]. Two groups of one-parameter transformation are usually found to give adequate treatment of boundary layer equations. $\dagger$ Each group gives rise to cases which will be separately discussed.

* The function $\psi$ is not strictly the stream function generally used in two-dimensional analyses since constant $\psi$-lines will not give actual flow streamlines. On the other hand, $\psi=$ const. does give projections of flow streamlines in the $x-y$ plane.

† The problem of systematically searching for all possible groups of transformation for a given system of partial differential equations has been the subject of a recent report by the authors [9] using Lie's theories of 
Case I. A one-parameter linear group of transformation is selected as

$$
\begin{aligned}
& x=A^{\alpha_{1}} \bar{x}, \quad y=A^{\alpha_{2}} \bar{y}, \quad \psi=A^{\alpha_{3}} \bar{\psi} \\
& w=A^{\alpha_{4}} \bar{w}, \quad U=A^{\alpha_{5}} \bar{U}, \quad W=A^{\alpha_{6}} \bar{W}
\end{aligned}
$$

where $\alpha_{1}, \alpha_{2}, \alpha_{3}, \alpha_{4}, \alpha_{5}, \alpha_{6}$, and $A$ are constants. We now seek relations among the $\alpha$ 's such that the basic equations will be invariant under this group of transformation. This can be achieved by substituting the transformation into equations (8) and (9). Thus, we obtain

$$
\begin{aligned}
& A^{2 \alpha_{3}-\alpha_{1}-2 \alpha_{2}}\left\{\frac{\partial \bar{\psi}}{\partial \bar{y}} \frac{\partial^{2} \bar{\psi}}{\partial \bar{x} \partial \bar{y}}-\frac{\partial \bar{\psi}}{\partial \bar{x}} \frac{\partial^{2} \bar{\psi}}{\partial \bar{y}^{2}}\right\} \\
& =\frac{\partial}{\partial \bar{y}}\left\{\left[A^{n \alpha_{3}-(2 n+1) \alpha_{2}}\left(\frac{\partial^{2} \bar{\psi}}{\partial \bar{y}^{2}}\right)^{2}+A^{(n-1) \alpha_{3}+\alpha_{4}-(n+2) \alpha_{2}}\left(\frac{\partial \bar{w}}{\partial \bar{y}}\right)^{2}\right]^{\frac{n-1}{2}} \frac{\partial^{2} \bar{\psi}}{\partial \bar{y}^{2}}\right\} \\
& +A^{2 \alpha_{5}-\alpha_{1}} \bar{U} \frac{\mathrm{d} \bar{U}}{\mathrm{~d} \bar{x}}
\end{aligned}
$$

and

$$
\begin{aligned}
& A^{\alpha_{3}+\alpha_{4}-\alpha_{1}-\alpha_{2}}\left\{\frac{\partial \bar{\psi}}{\partial \bar{y}} \frac{\partial \bar{w}}{\partial \bar{x}}-\frac{\partial \bar{\psi}}{\partial \bar{x}} \frac{\partial \bar{w}}{\partial \bar{y}}\right\} \\
& =\frac{\partial}{\partial \bar{y}}\left\{\left[A^{(n-1) \alpha_{3}+\alpha_{4}-2 n \alpha_{2}}\left(\frac{\partial^{2} \bar{\psi}}{\partial \bar{y}^{2}}\right)^{2}+A^{n \alpha_{4}-(n+1) \alpha_{2}}\left(\frac{\partial \bar{w}}{\partial \bar{y}}\right)^{2}\right]^{\frac{n-1}{2}} \frac{\partial \bar{w}}{\partial \bar{y}}\right\}+A^{\alpha_{5}+\alpha_{6}-\alpha_{1}} \bar{U} \frac{\mathrm{d} \bar{W}}{\mathrm{~d} \bar{x}}
\end{aligned}
$$

From equations (10) and (11), it is seen that if the basic equations are to be invariant under this group of transformation, the powers of $A$ in each term should be equal. Therefore, equations (10) and (11) give

$$
\begin{gathered}
2 \alpha_{3}-\alpha_{1}-2 \alpha_{2}=(n-1) \alpha_{3}+\alpha_{4}-2 n \alpha_{2}=n \alpha_{3}-(2 n+1) \alpha_{2}=2 \alpha_{5}-\alpha_{1} \\
\alpha_{3}+\alpha_{4}-\alpha_{1}-\alpha_{2}=n \alpha_{4}-(n+1) \alpha_{2}=(n-1) \alpha_{3}+\alpha_{4}-2 n \alpha_{2}=\alpha_{5}+\alpha_{6}-\alpha_{1}
\end{gathered}
$$

From equations (12) and (13), we get

$$
\begin{aligned}
& \frac{\alpha^{2}}{\alpha_{1}}=\frac{1+(n-2) \alpha}{n+1} \\
& \frac{\alpha^{3}}{\alpha_{1}}=\frac{1+(2 n-1) \alpha}{n+1} \\
& \frac{\alpha_{4}}{\alpha_{1}}=\frac{\alpha_{5}}{\alpha_{1}}=\frac{\alpha_{6}}{\alpha_{1}}=\alpha
\end{aligned}
$$

infinitesimal contact transformation groups. For two-dimensional, laminar boundary layer equations, the linear and the spiral groups are found to be the only two possible groups. For the present problem, we would expect that the same conclusion will be obtained. 
The next step in this method is to find the so-called "absolute invariants" under this group of transformation. Absolute invariants are functions having the same form before and after the transformation. It is noted that

$$
\begin{aligned}
\frac{y}{x^{\frac{1+(n-2) \alpha}{n+1}}} & =\frac{\bar{y}}{\bar{x}^{\frac{1+(n-2) \alpha}{n+1}}} \\
\frac{\psi}{x^{\frac{1+(2 n-1) \alpha}{n+1}}} & =\frac{\bar{\psi}}{\frac{1+(2 n-1) \alpha}{n+1}} \\
\frac{w}{x^{\alpha}} & =\frac{\bar{w}}{\bar{x}^{\alpha}} \\
\frac{U}{x^{\alpha}} & =\frac{\bar{U}}{\bar{x}^{\alpha}}
\end{aligned}
$$

and

$$
\frac{W}{x^{\alpha}}=\frac{\bar{W}}{\bar{x}^{\alpha}}
$$

Therefore, these functions are absolute invariants under this group of transformation. We therefore obtain the transformed independent and dependent variables as

$$
\begin{gathered}
\eta=\frac{y}{x^{\frac{1+(n-2) \alpha}{n+1}}} \\
F_{1}(\eta)=\frac{\psi}{x^{\frac{1+(2 n-1) \alpha}{n+1}}} \\
G_{1}(\eta)=\frac{w}{x^{\alpha}} \\
\bar{U}_{1}=\frac{\bar{U}}{x^{\alpha}}
\end{gathered}
$$

and

$$
\bar{W}_{1}=\frac{\bar{W}}{x^{\alpha}}
$$

Substituting for independent and dependent variables in equations (8) and (9) expressions found from equations (17)-(21) we expect to obtain a set of equations which are ordinary differential equations or very close to ordinary differential equations (see [4]).

Specifically we obtain:

$$
\begin{aligned}
\alpha\left(\frac{\mathrm{d} F_{1}}{\mathrm{~d} \eta}\right)^{2}-\frac{1+(2 n-1) \alpha}{n+1} F_{1} \frac{\mathrm{d}^{2} F_{1}}{\mathrm{~d} \eta^{2}} & \\
= & \frac{\mathrm{d}}{\mathrm{d} \eta}\left\{\left[\left(\frac{\mathrm{d}^{2} F_{1}}{\mathrm{~d} \eta^{2}}\right)^{2}+\left(\frac{\mathrm{d} G_{1}}{\mathrm{~d} \eta}\right)^{2}\right]^{\frac{n-1}{2}} \frac{\mathrm{d}^{2} F_{1}}{\mathrm{~d} \eta^{2}}\right\}+\alpha \bar{U}_{1}^{2}
\end{aligned}
$$




$$
\begin{aligned}
& \alpha G_{1} \frac{\mathrm{d} F_{1}}{\mathrm{~d} \eta}-\frac{1+(2 n-1) \alpha}{n+1} F_{1} \frac{\mathrm{d} G_{1}}{\mathrm{~d} \eta} \\
& \quad=\frac{\mathrm{d}}{\mathrm{d} \eta}\left\{\left[\left(\frac{\mathrm{d}^{2} F_{1}}{\mathrm{~d} \eta^{2}}\right)^{2}+\left(\frac{\mathrm{d} G_{1}}{\mathrm{~d} \eta}\right)^{2}\right]^{\frac{n-1}{2}} \frac{\mathrm{d} G_{1}}{\mathrm{~d} \eta}\right\}+\alpha \bar{U}_{1} \bar{W}_{1}
\end{aligned}
$$

which are ordinary differential equations if $U_{1}$, and $W_{1}$, are constants.

The conditions for the existence of similarity solutions can be obtained by considering the boundary conditions which must be constant. Thus, the only possible forms of $U(x)$ and $W(x)$ are

$$
\bar{U}(x)=C_{1} x^{\alpha}
$$

and

$$
\bar{W}(x)=C_{2} x^{\alpha}
$$

where $C_{1}$ and $C_{2}$ are arbitrary constants. For these forms of $U(x)$ and $W(x)$, equations (22) and (23) and their boundary conditions become

$$
\begin{aligned}
& \alpha\left(\frac{\mathrm{d} F_{1}}{\mathrm{~d} \eta}\right)^{2}-\frac{1+(2 n-1) \alpha}{n+1} F_{1} \frac{\mathrm{d}^{2} F_{1}}{\mathrm{~d} \eta^{2}} \\
& =\frac{\mathrm{d}}{\mathrm{d} \eta}\left\{\left[\left(\frac{\mathrm{d}^{2} F_{1}}{\mathrm{~d} \eta^{2}}\right)^{2}+\left(\frac{\mathrm{d} G_{1}}{\mathrm{~d} \eta}\right)^{2}\right]^{\frac{n-1}{2}} \frac{\mathrm{d}^{2} F_{1}}{\mathrm{~d} \eta^{2}}\right\}+\alpha C_{1}^{2} \\
& \alpha G_{1} \frac{\mathrm{d} F_{1}}{\mathrm{~d} \eta}-\frac{1+(2 n-1) \alpha}{n+1} F_{1} \frac{\mathrm{d} G_{1}}{\mathrm{~d} \eta} \\
& =\frac{\mathrm{d}}{\mathrm{d} \eta}\left\{\left[\left(\frac{\mathrm{d}^{2} F_{1}}{\mathrm{~d} \eta^{2}}\right)^{2}+\left(\frac{\mathrm{d} G_{1}}{\mathrm{~d} \eta}\right)^{2}\right]^{\frac{n-1}{2}} \frac{\mathrm{d} G_{1}}{\mathrm{~d} \eta}\right\}+\alpha C_{1} C_{2}
\end{aligned}
$$

with the boundary conditions

$$
\begin{array}{ll}
\eta=0: & F_{1}=\frac{\mathrm{d} F_{1}}{\mathrm{~d} \eta}=G_{1}=0 \\
\eta=\infty: & \frac{\mathrm{d} F_{1}}{\mathrm{~d} \eta}=\bar{U}_{1}=C_{1}, \quad G_{1}=\bar{W}_{1}=C_{2}
\end{array}
$$

Case II. A one-parameter spiral group of transformation is chosen in the form

$$
\begin{aligned}
x & =\beta_{1} b+\bar{x}, & y & =\mathrm{e}^{\beta_{2} b} \bar{y}, & \psi & =\mathrm{e}^{\beta_{3} b} \bar{\psi} \\
w & =\mathrm{e}^{\beta_{4} b} \bar{w}, & \bar{U} & =\mathrm{e}^{\beta_{5} b} \bar{U}, & \bar{W} & =\mathrm{e}^{\beta_{6} b} \bar{W}
\end{aligned}
$$

Following the same procedures as in Case $\mathrm{I}$, the following absolute invariants are obtained

$$
\begin{gathered}
\xi=\frac{y}{\exp \left(\frac{n-2}{n+1}\right) \beta x} \\
F_{2}(\xi)=\frac{\Psi}{\exp \left(\frac{2 n-1}{n+1}\right) \beta x}
\end{gathered}
$$




$$
\begin{aligned}
& G_{2}(\xi)=\frac{w^{\prime}}{\mathrm{e}^{\beta x}} \\
& \bar{U}_{2}=\frac{\bar{U}}{\mathrm{e}^{\beta x}}
\end{aligned}
$$

and

$$
\bar{W}_{2}=\frac{\bar{W}}{\mathrm{e}^{\bar{\beta} x}}
$$

where $\beta$ is an arbitrary constant. Since the mainstream velocities, $U$ and $W$, are assumed to be functions of $x$ only, we conclude that similarity solutions exist only for the special case

$$
\bar{U}(x)=C_{3} \mathrm{e}^{\beta x}
$$

and

$$
\bar{W}(x)=C_{4} \mathrm{e}^{\beta x}
$$

where $C_{3}$ and $C_{4}$ are arbitrary constants. The basic equations may then be transformed through the use of equations (28)-(32) to obtain:

$$
\begin{aligned}
\beta\left(\frac{\mathrm{d} F_{2}}{\mathrm{~d} \xi}\right)^{2}-\left(\frac{2 n-1}{n+1}\right) \beta F_{2} \frac{\mathrm{d}^{2} F_{2}}{\mathrm{~d} \xi^{2}} & \\
& =\frac{\mathrm{d}}{\mathrm{d} \xi}\left\{\left[\left(\frac{\mathrm{d}^{2} F_{2}}{\mathrm{~d} \xi^{2}}\right)^{2}+\left(\frac{\mathrm{d} G_{2}}{\mathrm{~d} \xi}\right)^{2}\right]^{\frac{n-1}{2}} \frac{\mathrm{d}^{2} F_{2}}{\mathrm{~d} \xi^{2}}\right\}+\beta C_{3}^{2}
\end{aligned}
$$

and

$$
\beta G_{2} \frac{\mathrm{d} F_{2}}{\mathrm{~d} \xi}-\left(\frac{2 n-1}{n+1}\right) \beta F_{2} \frac{\mathrm{d} G_{2}}{\mathrm{~d} \xi}
$$

with the boundary conditions

$$
=\frac{\mathrm{d}}{\mathrm{d} \xi}\left\{\left[\left(\frac{\mathrm{d}^{2} F_{2}}{\mathrm{~d} \xi^{2}}\right)^{2}+\left(\frac{\mathrm{d} G_{2}}{\mathrm{~d} \xi}\right)^{2}\right]^{\frac{n-1}{2}} \frac{\mathrm{d} G_{2}}{\mathrm{~d} \xi}\right\}+\beta C_{3} C_{4}
$$

$$
\begin{array}{ll}
\xi=0: & F_{2}=\frac{\mathrm{d} F_{2}}{\mathrm{~d} \xi}=G_{2}=0 \\
\xi=\infty: & \frac{\mathrm{d} F_{2}}{\mathrm{~d} \xi}=\bar{U}_{2}=C_{3}, \quad G_{2}=\bar{W}_{2}=C_{4}
\end{array}
$$

From the above analysis, it is seen that similarity solutions may exist for flows for which the mainstream velocities $U$ and $W$ are either powers or exponentials of $x$, as shown in equations (24), (25), (33) and (34). We also have

$$
\bar{U}(x)=\text { (Const.) } \bar{W}(x)
$$

This means the mainflow streamlines are straight lines. Although this is a severe restriction, the form of velocity components is somewhat more general than the cases found by Schowalter [4].*

* Schowalter apparently placed the restriction that all derivatives of velocities are non-vanishing. 


\section{SIMILARITY SOLUTIONS FOR SMALL CROSS FLOW}

If the cross-wise velocity is assumed to be small, then the restriction that

$$
\bar{U}(x)=\text { (const.) } \bar{W}(x)
$$

can be relaxed, and the mainflow streamlines need not be straight. Moreover, the momentum equation in the $z$-direction will be linear in $W$, and the principle of superposition of solutions may be applied.

The simplifications permitted from the assumption of small cross flow may be made evident by considering the limiting deflection angle, $\theta$, of the streamlines within the boundary layer. This angle is the arctangent of the ratio of velocities in the $z$ - and $x$-directions, evaluated at $y=0$, i.e.

$$
\tan \theta=\lim _{y \rightarrow 0} \frac{w}{u}=\lim _{y \rightarrow 0} \frac{\left(\frac{\partial w}{\partial y}\right)}{\left(\frac{\partial u}{\partial y}\right)}
$$

L'hospital's rule has been used because at $y=0$, both $w$ ' and $u$ are zero. Therefore, for small cross flow, i.e. small $\theta$, we would expect

$$
\frac{\partial w}{\partial y} \ll \frac{\partial u}{\partial y}
$$

within the boundary layer. The basic equations, equations (8) and (9), may then be simplified to

$$
\begin{aligned}
& \frac{\partial \psi}{\partial y} \frac{\partial^{2} \psi}{\partial x \partial y}-\frac{\partial \psi}{\partial x} \frac{\partial^{2} \psi}{\partial y^{2}}=\frac{\partial}{\partial y}\left(\frac{\partial^{2} \psi}{\partial y^{2}}\right)^{n}+\bar{U} \frac{\mathrm{d} \bar{U}}{\mathrm{~d} x} \\
& \frac{\partial \psi}{\partial y} \frac{\partial w}{\partial x}-\frac{\partial \psi}{\partial x} \frac{\partial w}{\partial y}=\frac{\partial}{\partial y}\left[\left(\frac{\partial^{2} \psi}{\partial y^{2}}\right)^{n-1} \frac{\partial w}{\partial y}\right]+\bar{U} \frac{\mathrm{d} \bar{W}}{\mathrm{~d} x}
\end{aligned}
$$

with the boundary conditions

$$
\begin{array}{ll}
y=0: & \frac{\partial \psi}{\partial x}=\frac{\partial \psi}{\partial y}=w=0 \\
y=\infty: & \frac{\partial \psi}{\partial y}=\bar{U}(x), w=\bar{W}(x)
\end{array}
$$

By following the same procedures as in the preceding section, the following results are obtained.

Case I. For the linear group of transformation, the absolute invariants are found to be

$$
\begin{gathered}
\eta=\frac{y}{x^{\frac{1+(n-2) y}{n+1}}} \\
F_{3}(\eta)=\frac{\psi}{x^{\frac{1+(2 n-1) y}{n+1}}}
\end{gathered}
$$




$$
\begin{gathered}
G_{3 i}(\eta)=\frac{w}{x^{i}} \\
\bar{U}_{3}=\frac{\bar{U}}{x^{\gamma}}
\end{gathered}
$$

and

$$
\bar{W}_{3}=\frac{\bar{W}}{x^{i}}
$$

where $\gamma$ and $i$ are constants.

By considering the boundary condition, we conclude that similarity solutions will exist if

$$
\bar{U}(x)=C_{5} x^{\gamma}
$$

and

$$
\bar{W}(x)=C_{6} x^{i}
$$

For these forms of $U$ and $W$, the basic equations (8) and (9), are transformed to

$$
\begin{gathered}
\gamma\left(\frac{\mathrm{d} F_{3}}{\mathrm{~d} \eta}\right)^{2}-\frac{1+(2 n-1) \gamma}{n+1} F_{3} \frac{\mathrm{d}^{2} F_{3}}{\mathrm{~d} \eta^{2}}=\frac{\mathrm{d}}{\mathrm{d} \eta}\left(\frac{\mathrm{d}^{2} F_{3}}{\mathrm{~d} \eta^{2}}\right)^{n}+\gamma C_{5}^{2} \\
i G_{3 i} \frac{\mathrm{d} F_{3}}{\mathrm{~d} \eta}-\frac{1+(2 n-1) \gamma}{n+1} F_{3} \frac{\mathrm{d} G_{3 i}}{\mathrm{~d} \eta}=\frac{\mathrm{d}}{\mathrm{d} \eta}\left\{\left(\frac{\mathrm{d}^{2} F_{3}}{\mathrm{~d} \eta^{2}}\right)^{n-1} \frac{\mathrm{d} G_{3 i}}{\mathrm{~d} \eta}\right\}+i C_{5} C_{6}
\end{gathered}
$$

with the boundary conditions

$$
\begin{aligned}
& \eta=0: \quad F_{3}=\frac{\mathrm{d} F_{3}}{\mathrm{~d} \eta}=G_{3 i}=0 \\
& \eta=\infty: \quad \frac{\mathrm{d} F_{3}}{\mathrm{~d} \eta}=\bar{U}_{3}=C_{5}, \quad G_{3 i}=\bar{W}_{3}=C_{6}
\end{aligned}
$$

being ordinary differential equations.

Equation (45) has the same form as equation (24), which means that the same form of mainstream velocity, $U$, for both cases. However, restrictions on the mainstream velocity in the $z$-direction, $W$, are considerably relaxed for the case of small cross flow, and $W$ need no longer equal $U$. Furthermore, equation (39) is linear, thus the principle of superposition can be applied, and the preceding result can be generalized at once. Assume that $W$ is given by

$$
\bar{W}(x)=\sum_{i} a_{i} X^{i}
$$

It may be verified by substitution that if $W_{i}$ denotes a solution of equation (39), then

$$
w=\sum_{i} w_{i}=\sum_{i} a_{i} x^{i} G_{3 i}(\eta)
$$

will be solution to equation (39) with $W$ given by equation (49). For the case of $U=$ $U_{3}=$ const., i.e. $\gamma=0$, and $n=1$, the above case is reduced to that analyzed by Hansen and Herzig [5] (although the limitation of small cross flow was not required). 
Case II. For the spiral group of transformation, the following absolute invariants are obtained.

$$
\begin{gathered}
\xi=\frac{y}{\exp \frac{n-2}{n+1} s x} \\
F_{4}(\xi)=\frac{\psi}{\exp \frac{2 n-1}{n+1} s x} \\
G_{4 j}(\xi)=\frac{w}{\mathrm{e}^{j x}} \\
\bar{U}_{4}=\frac{\bar{U}}{\mathrm{e}^{s x}}
\end{gathered}
$$

and

$$
\bar{W}_{4}=\frac{\bar{W}}{\mathrm{e}^{j x}}
$$

Again, the conditions for the existence of similarity solutions are obtained from the boundary conditions. It is found that similarity solutions will exist if

$$
\bar{U}(x)=C_{7} \mathrm{e}^{s x}
$$

and

$$
\bar{W}(x)=C_{8} \mathrm{e}^{j x}
$$

The basic equations, equations (8) and (9), then become

$$
\begin{gathered}
s\left(\frac{\mathrm{d} F_{4}}{\mathrm{~d} \xi}\right)^{2}-\left(\frac{2 n-1}{n+1}\right) s F_{4} \frac{\mathrm{d}^{2} F_{4}}{\mathrm{~d} \xi^{2}}=\frac{\mathrm{d}}{\mathrm{d} \xi}\left(\frac{\mathrm{d}^{2} F_{4}}{\mathrm{~d} \xi^{2}}\right)^{n}+s C_{7}^{2} \\
j G_{4 j} \frac{\mathrm{d} F_{4}}{\mathrm{~d} \xi}-\left(\frac{2 n-1}{n+1}\right) s F_{4} \frac{\mathrm{d} G_{4 j}}{\mathrm{~d} \xi}=\frac{\mathrm{d}}{\mathrm{d} \xi}\left[\left(\frac{\mathrm{d}^{2} F_{4}}{\mathrm{~d} \xi^{2}}\right)^{n-1} \frac{\mathrm{d} G_{4 j}}{\mathrm{~d} \xi}\right]+j C_{7} C_{8}
\end{gathered}
$$

with the boundary conditions

$$
\begin{aligned}
\xi=0: & F_{4}=\frac{\mathrm{d} F_{4}}{\mathrm{~d} \xi}=G_{4 j}=0 \\
\xi=\infty: & \frac{\mathrm{d} F_{4}}{\mathrm{~d} \xi}=\bar{U}_{4}=C_{7}, \quad G_{4 j}=\bar{W}_{4}=C_{8}
\end{aligned}
$$

being independent of $x$ and $y$.

It is seen from the preceding analysis that for cases with small cross flow, the boundary layer equations can be solved exactly by similarity transformation if the mainstream velocity is in the form of equation (50). Since the coefficients in equation (50) are arbitrary, various streamline shapes can be constructed by proper choice of these coefficients. A particular shape (e.g. the contour of a channel wall) may be approximated by a polynominal 
according to usual numerical procedures. Thus, the class of flows becomes a useful tool in investigating the influence of various streamline configurations on boundary layer behavior.

\title{
CONCLUSIONS
}

The analysis of the laminar, incompressible three-dimensional boundary layer equations of power-law fluids with streamlines forming a system of "Translates" led to solutions for mainstream flows described by equations (24), (25), (33) and (34). By placing the condition of small cross flow, restrictions on the mainstream velocity $W$ are considerably relaxed, as is shown in equations (45), (46), (56) and (57). Furthermore, the linearity of the momentum equation in the crosswise direction makes it possible to generalize the form to any mainstream shape which can be approximated by a polynomial (e.g. the contour of a channel wall).

Numerical solutions of the ordinary differential equations for different values of the parameters will be given in a later paper.

\section{REFERENCES}

[1] A. ACRIvos, A theoretical analysis of laminar natural convection heat transfer to non-Newtonian fluids. A.I.Ch.E. Jl 6, 584 (1960).

[2] A. ACrivos, M. J. Shah and E. E. Petrrson, Momentum and heat transfer in laminar boundary-layer flows of non-Newtonian fluids past external surfaces. A.I.Ch.E. Jl 6, 312 (1960).

[3] J. N. KapUR and R. C. SRIvastava, Similar solutions of the boundary layer equations for power law fluids. Z. angew. Math. Phys. 14, 383 (1963).

[4] W. R. SCHOWALTER, The application of boundary-layer theory to power law pseudoplastic fluids: similar solutions. A.I.Ch.E. Jl 6, 24 (1960).

[5] A. G. HANSEN and H. Z. Herzig, Cross flows in laminar, incompressible, boundary layers. NACA TN 3651 (1955).

[6] G. BiRKHOFF, Mydrodynamics. Dover (1955).

[7] A. J. A. Morgan, Possible similarity solutions of the laminar, incompressible, boundary layer equations. [Discussion]. Trans. Am. Soc. mech. Engrs 80, 1559 (1958).

[8] A. G. HANSEN, Similarity Analyses of Boundary Value Problems in Engineering. Prentice-Hall (1965).

[9] T. Y. NA, D. E. ABbotT and A. G. HANSEN, Similarity analysis of partial differential equations. Technical Report, NASA contract 8-20065, University of Michigan, Ann Arbor, Mich. (April 1967).

(Received 18 February 1966; revised 11 March 1967)

\begin{abstract}
Résumé- On analyse la possibilité de trouver des solutions de similitude aux équations, dans un système de coordonnées trirectangulaires, de la couche limite d'un fluide incompressible en mouvement permanent obéissant à une fonction puissance. On trouve qu'en général les deux composantes du flux principal doivent différer par au plus une constante multiplicative et que ces composantes sont des puissances ou des exponentielles de la coordonnée de la direction du flux direct.

En supposant que les flux transversaux sont petits, la composante transversale peut être généralisée et on trouve qu'elle est représentée par un polynôme dans la variable du flux direct.
\end{abstract}

Zusammenfassung-Es wird die Möglichkeit untersucht, Ähnlichketslösungen zu den dreidimensionalen, stationären, inkompressiblen Grenzschichtsgleichungen in rechtwinkligen Koordinaten für eine Flüssigkeit zu finden, die einem Potenzgesetz unterliegt. Es ergibt sich, dass sich die zwei Komponenten der Hauptströmung im allgemeinen durch höchstens eine multiplikative Konstante unterscheiden müssen, und dass diese Komponenten Potenz-oder Exponentialfunktionen der Koordinate darstellen, die in die Durchstromungsrichtung weist.

Durch die Annahme kleiner Kreuzströmung kann die Kreuzströmungskomponente verallgemeinert werden. Sie lässt sich dann durch ein Polynom in der Durchflüssvariablen darstellen. 
Абстракт-Делается аналия возможности разыскания решений подобия для уравнений трехмерного, стационарного, несжимаемого пограничного слон в прямоугольных координатах для жидкости подчиняющейся степенному закону. Установлено, что вообще, две компоненты главного потока течения могут отличатсья друг от друга не более чем мультипликативной константой и что әти константы являются степенными или показательными функциями координат в направлении "сквозного течения".

В предположении малых поперечных течений, можно обобщить компоненту поперечного течения представляя ее в виде функции от переменной сквозного течения. 